PROCEEDINGS OF THE

AMERICAN MATHEMATICAL SOCIETY

Volume 132, Number 1, Pages 59-65

S 0002-9939(03)07008-4

Article electronically published on June 5, 2003

\title{
RECOGNITION OF SUBGROUPS OF DIRECT PRODUCTS OF HYPERBOLIC GROUPS
}

\author{
MARTIN R. BRIDSON AND CHARLES F. MILLER III
}

(Communicated by Stephen D. Smith)

\begin{abstract}
We give examples of direct products of three hyperbolic groups in which there cannot exist an algorithm to decide which finitely presented subgroups are isomorphic.
\end{abstract}

In [1] Baumslag, Short and the present authors constructed an example of a biautomatic group $G$ in which there is no algorithm that decides isomorphism among the finitely presented subgroups of $G$. The group $G$ was a direct product of a certain (word) hyperbolic group $H$ with an HNN extension $B$ of $H$; the group $B$ was CAT(0) and biautomatic but not hyperbolic. At the time of writing [1] we were unable to construct, more simply, a direct product of hyperbolic groups in which the isomorphism problem for finitely presented subgroups was unsolvable.

In the course of our project on finiteness properties of subdirect products [7], we uncovered a new criterion for the finite presentability of certain semidirect products (see Section 2). That, combined with an improved understanding of subgroups of direct products, has enabled us to prove the following result:

Theorem 1. Let $1 \rightarrow K \rightarrow \Gamma \rightarrow L \rightarrow 1$ be an exact sequence of groups. Suppose that

(1) $\Gamma$ is torsion-free and (word) hyperbolic,

(2) $K$ is infinite and finitely generated, and

(3) $L$ is a non-abelian free group.

If $F$ is a non-abelian free group, then there is no algorithm to decide which pairs of finitely presented subgroups of $\Gamma \times \Gamma \times F$ are isomorphic.

In more detail, there is a recursive sequence $\Delta_{i}$, where $i=0,1, \ldots$, of finite subsets of $\Gamma \times \Gamma \times F$ together with finite presentations $\left\langle\Delta_{i} \mid \Theta_{i}\right\rangle$ of the subgroups they generate such that there is no algorithm to determine whether or not $\left\langle\Delta_{i} \mid \Theta_{i}\right\rangle \cong$ $\left\langle\Delta_{0} \mid \Theta_{0}\right\rangle$.

An example of such a group $\Gamma$ is given, for instance, by applying the construction of Rips [14 to a non-abelian free group.

Received by the editors January 31, 2002 and, in revised form, September 9, 2002.

2000 Mathematics Subject Classification. Primary 20F10, 20F67.

Key words and phrases. Hyperbolic groups, finitely presented subgroups, isomorphism problem.

The first author's research was funded by an Advanced Fellowship from the EPSRC. 
The crucial element of undecidability in Theorem 1 comes from an encoding of the fact that the generation problem for a direct product of non-abelian free groups is undecidable.

It is possible to avoid using the criteria we develop for finite presentability of certain semidirect products by appealing to recent deeper results of Mosher and others as discussed in Section 4.

\section{A CRITERION FOR FINITE PRESENTABILITy}

Recall that a subgroup $H$ of a direct product $A \times B$ is termed a subdirect product if its projection to each of $A$ and $B$ is surjective.

We discovered the following criterion in the course of our project on finiteness properties of subdirect products [7. It is based on the special case proved in Lemma 2.3 of $[5]$.

Proposition 2. Let $K_{1}$ and $K_{2}$ be finitely generated groups. Let $L_{1}$ and $L_{2}$ be finitely generated free groups and let $\phi_{i}: L_{i} \rightarrow$ Aut $K_{i}$ be homomorphisms such that the semidirect products $K_{i} \rtimes_{\phi_{i}} L_{i}$ are finitely presented.

Let $p: F \rightarrow H$ be a homomorphism from a finitely presented group $F$ to a subdirect product $H \subset L_{1} \times L_{2}$. Let $\Phi=\left(\phi_{1}, \phi_{2}\right) \circ p$. Then the associated semidirect product $\left(K_{1} \times K_{2}\right) \rtimes_{\Phi} F$ is finitely presented.

Proof. For each $i=1,2$, fix a finite set of generators $\mathcal{B}_{i}$ for $K_{i}$ and a free basis $\mathcal{C}_{i}$ for $L_{i}$. Now $K_{i}$ has a presentation of the form $\left\langle\mathcal{B}_{i} \mid \mathcal{T}_{i}\right\rangle$ where $\mathcal{T}_{i}$ consists of words in $\mathcal{B}_{i}$. Let $\mathcal{R}_{i}$ be the (finite) set of words $c^{-1} b c \beta^{-1}$ with $b \in \mathcal{B}_{i}$ and $c \in \mathcal{C}_{i}$, where $\beta$ is a word in $\mathcal{B}_{i}$ chosen so that $\beta=\left(\phi_{i}(c)\right)(b)$ in $K_{i}$.

With these choices, $\left\langle\mathcal{B}_{i}, \mathcal{C}_{i} \mid \mathcal{T}_{i}, \mathcal{R}_{i}\right\rangle$ is a presentation of $K_{i} \rtimes_{\phi_{i}} L_{i}$. Now by assumption $K_{i} \rtimes_{\phi_{i}} L_{i}$ is finitely presented. So for some finite subset $\mathcal{S}_{i} \subseteq \mathcal{T}_{i}$, the "subpresentation" $\left\langle\mathcal{B}_{i}, \mathcal{C}_{i} \mid \mathcal{S}_{i}, \mathcal{R}_{i}\right\rangle$ is a presentation for $K_{i} \rtimes_{\phi_{i}} L_{i}$, and all the remaining relations in $\mathcal{T}_{i}$ are consequences of those in $\mathcal{S}_{i}$ and $\mathcal{R}_{i}$.

Let $Z_{B}$ consist of the commutators $\left[b_{1}, b_{2}\right]$ with $b_{i} \in \mathcal{B}_{i}$. Then $\left\langle\mathcal{B}_{1}, \mathcal{B}_{2} \mid \mathcal{T}_{1}, \mathcal{T}_{2}, Z_{B}\right\rangle$ is a presentation for $K_{1} \times K_{2}$.

Choose a finite presentation $\langle X \mid Y\rangle$ for $F$. For each $i=1,2$, let $\mathcal{U}_{i}$ consist of words $x^{-1} b x \gamma^{-1}$ where $b \in \mathcal{B}_{i}$ and $x \in X$ and where the $\gamma=\left(\phi_{i}(p(x))\right)(b)$ are words in $\mathcal{B}_{i}$. It follows that

$$
\left\langle\mathcal{B}_{1}, \mathcal{B}_{2}, X \mid \mathcal{T}_{1}, \mathcal{T}_{2}, Z_{B}, Y, \mathcal{U}_{1}, \mathcal{U}_{2}\right\rangle
$$

is a presentation for $\left(K_{1} \times K_{2}\right) \rtimes_{\Phi} F$.

For each $c \in \mathcal{C}_{i}, i=1,2$, there is a word $\rho$ on $X$ such that $\rho \mapsto c$ under the composition $F \rightarrow L_{1} \times L_{2} \rightarrow L_{i}$. (This is the point at which we are using the fact that the image of $F$ is a subdirect product of $L_{1} \times L_{2}$.) Let $\mathcal{V}_{i}$ be the (finite) set of words $c \rho^{-1}$. Applying a simple Tietze transformation, it follows that

$$
\left\langle\mathcal{B}_{1}, \mathcal{B}_{2}, \mathcal{C}_{1}, \mathcal{C}_{2}, X \mid \mathcal{T}_{1}, \mathcal{T}_{2}, Z_{B}, Y, \mathcal{U}_{1}, \mathcal{U}_{2}, \mathcal{V}_{1}, \mathcal{V}_{2}\right\rangle
$$

is also a presentation for $\left(K_{1} \times K_{2}\right) \rtimes_{\Phi} F$ since the added relations $\mathcal{V}_{i}$ say that the added generators $c \in \mathcal{C}_{i}$ are abbreviations for the corresponding words $\rho$.

By construction, the finite sets of relations $\mathcal{R}_{i}$ are consequences of the relations in the above presentation. Applying a simple Tietze transformation we can therefore add them to obtain a new presentation

$$
\left\langle\mathcal{B}_{1}, \mathcal{B}_{2}, \mathcal{C}_{1}, \mathcal{C}_{2}, X \mid \mathcal{T}_{1}, \mathcal{T}_{2}, \mathcal{R}_{1}, \mathcal{R}_{2}, Z_{B}, Y, \mathcal{U}_{1}, \mathcal{U}_{2}, \mathcal{V}_{1}, \mathcal{V}_{2}\right\rangle
$$


for the group $\left(K_{1} \times K_{2}\right) \rtimes_{\Phi} F$.

Recall that the relations $\mathcal{T}_{i}$ are consequences of the finite set of relations $\mathcal{S}_{i} \cup \mathcal{R}_{i}$. So again applying a Tietze transformation we obtain the finite presentation

$$
\left\langle\mathcal{B}_{1}, \mathcal{B}_{2}, \mathcal{C}_{1}, \mathcal{C}_{2}, X \mid \mathcal{S}_{1}, \mathcal{S}_{2}, \mathcal{R}_{1}, \mathcal{R}_{2}, Z_{B}, Y, \mathcal{U}_{1}, \mathcal{U}_{2}, \mathcal{V}_{1}, \mathcal{V}_{2}\right\rangle
$$

for the group $\left(K_{1} \times K_{2}\right) \rtimes_{\Phi} F$. This completes the proof of the proposition.

\section{A SEED OF UNDECIDABILITY}

In the published version of his thesis (10, also 9], page 194, and 11]), the second author proved that the generation problem for the direct product $L \times L$ of two non-abelian, finitely generated free groups is unsolvable. Combining that construction with non-finite presentability results of Grunewald [8] or of Baumslag and Roseblade 2, we have the following:

Theorem 3. Let $L$ be a free group of rank at least 2 and let $X$ be a finite generating set for $L \times L$. For some sufficiently large integer $m$, there exists a recursive sequence $\Lambda_{n}$ of finite sets of words on $X \cup X^{-1}$ such that

(1) each $\Lambda_{n}$ has cardinality $m$;

(2) each of the subgroups $\left\langle\Lambda_{n}\right\rangle \subset L \times L$ is a subdirect product;

(3) $\left\langle\Lambda_{0}\right\rangle=L \times L$;

(4) there is no algorithm to determine whether or not $\left\langle\Lambda_{n}\right\rangle=L \times L$;

(5) if $\left\langle\Lambda_{n}\right\rangle \neq L \times L$, then $\left\langle\Lambda_{n}\right\rangle$ is not finitely presented.

It is convenient (though this could be avoided) to recall a bit more detail about these sets of words $\Lambda_{n}$. Suppose that $L$ has a free basis $c_{1}, \ldots, c_{k}$. The construction of $\Lambda_{n}$ involves a family of finite presentations of quotients of $L$ of the form $\Pi_{n}=$ $\left\langle c_{1}, \ldots, c_{k} \mid q_{n, 1}, \ldots, q_{n, m-k}\right\rangle$ all having the same set of generators and the same number $m-k$ of relators, the form of which depends on a parameter. The $m$ words in $\Lambda_{n}$ correspond to the elements

$$
\left\{\left(c_{1}, c_{1}\right), \ldots,\left(c_{k}, c_{k}\right),\left(q_{n, 1}, 1\right), \ldots,\left(q_{n, m-k}, 1\right)\right\}
$$

thought of as pairs in the direct product $L \times L$. The presentations $\Pi_{n}$ are chosen so that determining whether the group presented is trivial or infinite is an unsolvable problem. The subgroup generated by $\Lambda_{n}$ is all of $L \times L$ if and only if the group presented by $\Pi_{n}$ is trivial.

Beginning the proof of Theorem 1, Let $F$ be a free group of ranl] $2 m$ with basis $\left\{x_{1}, \ldots, x_{m}, y_{1}, \ldots, y_{m}\right\}$. If we list the elements of the sets $\Lambda_{n}$ as say $\Lambda_{n}=\left\{\lambda_{n, 1}, \ldots, \lambda_{n, m}\right\}$, ordered in consonance with (1), then we can define a homomorphism $p_{n}: F \rightarrow L \times L$ by $p_{n}\left(x_{i}\right)=\lambda_{n, i}$ and $p_{n}\left(y_{i}\right)=1$ for $i=1, \ldots, m$. Of course the image $p_{n}(F)=\left\langle\Lambda_{n}\right\rangle$.

We take $K=K_{i}$ and $L=L_{i}$ as in Proposition 2, Thus $\Gamma=\Gamma_{i}:=K_{i} \rtimes_{\phi_{i}} L_{i}$. Associated to each $\Lambda_{n}$ we have a semidirect product

$$
E_{n}=(K \times K) \rtimes_{\Phi_{n}} F .
$$

\footnotetext{
${ }^{1}$ Since a non-abelian free group contains a free group of any given finite rank, this suffices in order to prove Theorem 1.
} 
Suppose $b_{1}, \ldots, b_{p}$ generate $K$. Then, using the detailed notation above, $E_{n}$ is isomorphic to the subgroup of $\Gamma \times \Gamma \times F$ generated by the following set of elements:

$$
\begin{gathered}
\Delta_{n}=\left\{\left(b_{1}, 1,1\right), \ldots,\left(b_{p}, 1,1\right),\left(1, b_{1}, 1\right), \ldots,\left(1, b_{p}, 1\right),\right. \\
\left(c_{1}, c_{1}, x_{1}\right), \ldots,\left(c_{k}, c_{k}, x_{k}\right),\left(q_{n, 1}, 1, x_{k+1}\right), \ldots,\left(q_{n, m-k}, 1, x_{m}\right), \\
\left.\left(1,1, y_{1}\right), \ldots,\left(1,1, y_{m}\right)\right\} .
\end{gathered}
$$

Since each $\left\langle\Lambda_{n}\right\rangle \subset L \times L$ is a subdirect product, the groups $E_{n}$ are all finitely presented. Because $\left\{x_{1}, \ldots, x_{k}\right\}$ projects onto a basis for each direct factor $L$, the finite presentation for $E_{n}$ given in the proof of Proposition 2 easily simplifies to $\left\langle\Delta_{n} \mid \Theta_{n}\right\rangle=\left\langle\mathcal{B}_{1}, \mathcal{B}_{2}, X \mid \mathcal{S}_{1}, \mathcal{S}_{2}, \mathcal{U}_{n}\right\rangle$ where the $\mathcal{S}_{i}$ are fixed and the $\mathcal{U}_{n}$ can be written down explicitly using the words $q_{i, j}$.

Note that $E_{n}$ is a subdirect product of $\Gamma \times \Gamma \times F$. The structure of $E_{n}$ will obviously depend on $\Phi_{n}$ and hence $\Lambda_{n}$. If $\left\langle\Lambda_{n}\right\rangle=\left\langle\Lambda_{0}\right\rangle=L \times L$, then one is tempted to say that $E_{n}$ is "obviously" isomorphic to $E_{0}$, since the images of $\Phi_{n}$ and $\Phi_{0}$ are the same. But a little care is needed, since in general a semidirect product $G \rtimes_{\psi} F$ is determined by the actual map $\psi: F \rightarrow$ Aut $G$ but not by the set $\operatorname{im} \psi$. The issue here is one of Nielsen equivalence between different generating sets of $\operatorname{im} \psi$. It is to circumvent this difficulty that we took $F$ above to be a free group of rank $2 m$ rather than simply $m$. This apparent redundancy has been employed so that we can appeal to the following version of Rapaport's Theorem [13], which is proved is Section 4 of [6].

Lemma 4. Let $\Gamma$ be a group. Let $F_{2 m}$ be a free group of rank $2 m$ with basis $\left\{x_{1}, \ldots, x_{m}, y_{1}, \ldots, y_{m}\right\}$. Let $\phi_{1}, \phi_{2}: F_{2 m} \rightarrow \Gamma$ be homomorphisms such that $\operatorname{im} \phi_{1}=\operatorname{im} \phi_{2}$ and $\phi_{1}\left(y_{i}\right)=\phi_{2}\left(y_{i}\right)=1$ for $i=1, \ldots, m$. Then there exists an automorphism $\Psi: F_{2 m} \rightarrow F_{2 m}$ such that $\phi_{2} \circ \Psi=\phi_{1}$.

Corollary 5. If $\Gamma \subset$ Aut $N$, then under the above hypotheses $N \rtimes_{\phi_{1}} F_{2 m} \cong$ $N \rtimes_{\phi_{2}} F_{2 m}$.

Proof. Define $N \rtimes_{\phi_{1}} F_{2 m} \rightarrow N \rtimes_{\phi_{2}} F_{2 m}$ to be the identity on $N$ and $\Psi$ on $F_{2 m}$.

Corollary 6. In the above construction, if $\left\langle\Lambda_{n}\right\rangle=L \times L$, that is, if $p_{n}$ maps $F$ onto $L \times L$, then $E_{n} \cong E_{0}$; equivalently, $\left\langle\Delta_{n} \mid \Theta_{n}\right\rangle \cong\left\langle\Delta_{0} \mid \Theta_{0}\right\rangle$.

To complete the proof of Theorem 11 we need a method of showing that if $\left\langle\Lambda_{n}\right\rangle \neq L \times L$, then $\left\langle\Delta_{n} \mid \Theta_{n}\right\rangle \approx\left\langle\Delta_{0} \mid \Theta_{0}\right\rangle$. This is accomplished in the next section by considering centralizers of pairs of elements.

\section{Centralizers in Full Subgroups}

If $\Gamma$ is a torsion-free hyperbolic group, then the centralizer of each non-trivial element $\gamma \in \Gamma$ is the cyclic group $\langle\bar{\gamma}\rangle$, where $\bar{\gamma}$ is the maximal root of $\gamma$. It follows that if $\gamma_{1}$ and $\gamma_{2}$ are two non-commuting elements of $\Gamma$, then the centralizer $C_{\Gamma}\left(\gamma_{1}, \gamma_{2}\right)$ of the pair is trivial.

Let $D=\Gamma_{1} \times \cdots \times \Gamma_{n}$ be a direct product of non-cyclic, torsion-free hyperbolic groups. Consider a non-commuting pair of elements $x=\left(x_{1}, \ldots, x_{n}\right)$ and $y=$ $\left(y_{1}, \ldots, y_{n}\right)$ of $D$. Then for some $i$, their coordinates $x_{i}$ and $y_{i}$ do not commute. If $z=\left(z_{1}, \ldots, z_{n}\right)$ commutes with both $x$ and $y$, then $z_{i}=1$ since $\Gamma_{i}$ is torsion-free hyperbolic. Thus the elements in the centralizer of the pair $C_{D}(x, y)$ all have their $i$-th coordinates equal to 1 , and so

$$
C_{D}(x, y) \leq \Gamma_{1} \times \cdots \times \Gamma_{i-1} \times 1 \times \Gamma_{i+1} \times \cdots \times \Gamma_{n}
$$


Definition 1. A subgroup $H$ of a direct product $\Gamma_{1} \times \cdots \times \Gamma_{n}$ is said to be full if $H \cap \Gamma_{i}$ contains a non-commuting pair of elements $x_{i}, y_{i}$ for each $i=1, \ldots, n$.

Suppose that $H$ is a full subgroup of $D$. Then the collection $\Sigma$ of centralizers of non-commuting pairs of elements of $H$ has a finite set of maximal elements, namely the centralizers of pairs $x_{i}$ and $y_{i}$ which are non-commuting pairs in $H \cap \Gamma_{i}$. These maximal elements are exactly the subgroups

$$
M_{i}=H \cap\left(\Gamma_{1} \times \cdots \times \Gamma_{i-1} \times 1 \times \Gamma_{i+1} \times \cdots \times \Gamma_{n}\right)
$$

for $i=1, \ldots, n$. The set of subgroups $\left\{M_{1}, \ldots, M_{n}\right\}$ form an isomorphism invariant of $H$. So, if $\phi: H \rightarrow K$ is an isomorphism, then each $\phi\left(M_{i}\right)$ will be a maximal centralizer of a non-commuting pair of elements of $K$.

A set of subgroups of $H$ is said to be characteristic if any automorphism of $H$ permutes the subgroups in the set. Clearly $\left\{M_{1}, \ldots, M_{n}\right\}$ is also a characteristic set of subgroups of $H$. Now each of the subgroups $H \cap \Gamma_{i}$ is the intersection of the $n-1$ distinct $M_{j}$ which contain it. It follows that the $H \cap \Gamma_{i}$ also form a characteristic set of subgroups of $H$. From these observations we conclude the following:

Proposition 7. If $H$ is a full subgroup of a direct product $D=\Gamma_{1} \times \cdots \times \Gamma_{n}$ of non-cyclic, torsion-free hyperbolic groups, then the set of subgroups $\left\{H \cap \Gamma_{i}\right\}$ is characteristic, and hence

$$
\left(H \cap \Gamma_{1}\right) \times \cdots \times\left(H \cap \Gamma_{n}\right)
$$

is a characteristic subgroup.

Applying these considerations to the case $H$ is $D$ itself, we obtain the following:

Corollary 8. If $D=\Gamma_{1} \times \cdots \times \Gamma_{n}$ is the direct product of non-cyclic, torsion-free hyperbolic groups, then the set of subgroups $\left\{\Gamma_{i}\right\}$ is characteristic. In particular, the decomposition of $D$ as a direct product of hyperbolic subgroups is unique.

Observe that the corollary is no longer true if either cyclic or finite factors are allowed in the definition of $D$ as a direct sum of hyperbolic groups.

Completing the proof of Theorem 1. We resume the notation developed in Section 2. Since $K$ is an infinite normal subgroup of the non-cyclic torsion-free hyperbolic group $\Gamma, K$ is non-abelian and so contains a pair of non-commuting elements. The intersections of $\left\langle\Delta_{n}\right\rangle$ with the first and second factors of $\Gamma_{1} \times \Gamma_{2} \times F$ (where we have added subscripts to distinguish the factors) are just two copies $K_{1}$ and $K_{2}$ of $K$. In particular they are finitely generated.

The intersection of $\left\langle\Delta_{n}\right\rangle$ with the third factor of $\Gamma \times \Gamma \times F$ is the kernel of $p_{n}: F \rightarrow L \times L$. The image of $p_{n}$ is $\left\langle\Lambda_{n}\right\rangle \subseteq L \times L$. So, if $\left\langle\Lambda_{n}\right\rangle=L \times L$, then ker $p_{n}=F \cap\left\langle\Delta_{n}\right\rangle$ is finitely normally generated, since $L \times L$ is finitely presented. In particular, $\operatorname{ker} p_{0}=F \cap\left\langle\Delta_{0}\right\rangle$ is finitely normally generated.

On the other hand, if $p_{n}(F)=\left\langle\Lambda_{n}\right\rangle \neq L \times L$, then $\operatorname{ker} p_{n}=F \cap\left\langle\Delta_{n}\right\rangle$ is not finitely normally generated, since $\left\langle\Lambda_{n}\right\rangle$ is not finitely presented.

We know from Proposition 7 that $\left\{K_{1}, K_{2}, F \cap\left\langle\Delta_{n}\right\rangle\right\}$ is a characteristic set of subgroups of $\left\langle\Delta_{n}\right\rangle$. The foregoing discussion tells us that all three of these subgroups will be finitely normally generated if and only if $\left\langle\Delta_{n}\right\rangle=L \times L$. Thus $\left\langle\Lambda_{n}\right\rangle \neq L \times L$ implies $\left\langle\Delta_{n} \mid \Theta_{n}\right\rangle \neq\left\langle\Delta_{0} \mid \Theta_{0}\right\rangle$. By Theorem 3 there is no algorithm to determine whether or not $\left\langle\Lambda_{n}\right\rangle=L \times L$. Therefore, deciding whether or not $\left\langle\Delta_{n} \mid \Theta_{n}\right\rangle \cong\left\langle\Delta_{0} \mid \Theta_{0}\right\rangle$ is an unsolvable problem. This completes the proof of Theorem 1 
Remark 1. A slight variation on the above proof shows that there is no algorithm to determine which finitely presented subgroups of $\Gamma \times \Gamma \times F$ are abstractly commensurable.

\section{HyPERBOLIC-BY-HYPERBOLIC HYPERBOLIC GROUPS}

As mentioned in the introduction, examples of hyperbolic groups $\Gamma$ as required for Theorem 1 are provided by the well-known construction of Rips [14]. If the subgroup $K$ in the theorem is also finitely presented, then the groups $E_{n}$ are visibly finitely presented without appeal to Section 1 . In this section we describe short exact sequences of torsion-free non-elementary hyperbolic groups, thus animating Theorem 1 with additional examples.

First we describe Mosher's examples [12. Let $S$ be a closed hyperbolic surface, pick a base point $p \in S$ and let $\Sigma=\pi_{1}(S, p)$ be the fundamental group. Let $\operatorname{MCG}(S)$ denote the mapping class group of $S$ (isotopy classes of self-homeomorphisms) and let $\operatorname{MCG}(S, p)$ be the mapping class group of $S$ punctured at $p$. The kernel of the natural map $\operatorname{MCG}(S, p) \rightarrow \operatorname{MCG}(S)$ is generated by homeomorphisms that drag the puncture $p$ around a simple closed loop in $\Sigma$ and are the identity off a neighborhood of the loop. This description gives an obvious identification of the kernel with $\Sigma$. Thus we have a short exact sequence

$$
1 \rightarrow \Sigma \rightarrow \operatorname{MCG}(S, p) \rightarrow \operatorname{MCG}(S) \rightarrow 1
$$

Thurston shows that the action of any pseudo-Anosov element $\phi \in \operatorname{MCG}(S)$ on the boundary of Teichmüller space has two fixed points. One says that a set of pseudo-Anosov mapping classes is independent if the fixed point sets of its elements are disjoint. It is not difficult to construct arbitrarily large independent sets of pseudo-Anosov mapping classes.

Theorem 9 (Mosher). Let $S$ be a closed hyperbolic surface. Suppose that $\phi_{1}, \ldots, \phi_{m}$ $\in \operatorname{MCG}(S)$ is an independent set of pseudo-Anosov mapping classes of $S$. Let $i_{1}, \ldots, i_{m}$ be positive integers, and consider the subgroup $H=\left\langle\phi_{1}^{i_{1}}, \ldots, \phi_{m}^{i_{m}}\right\rangle$ of $\operatorname{MCG}(S)$. Let $G \leq \operatorname{MCG}(S, p)$ be the preimage of $H$. If $i_{1}, \ldots, i_{m}$ are sufficiently large, then the group $H$ is free on the given generators and $G$ is word hyperbolic. Therefore $1 \rightarrow K \rightarrow G \rightarrow H \rightarrow 1$ is a short exact sequence of non-elementary hyperbolic groups.

Hyperbolic groups of the form $\Gamma=F \rtimes F$, with $F$ free, have been constructed by Bestvina, Feighn and Handel in analogy with the above construction [3]. Brady and Miller [4 have shown that such groups arise as the fundamental groups of certain compact, negatively curved 2-complexes $X$. In this case $\Gamma \times \Gamma \times F$ is the fundamental group of $Z=X \times X \times Y$, where $Y$ is a compact metric graph. In the product metric $Z$ is non-positively curved in the sense of A. D. Alexandrov, and we can therefore recover Theorem B of [1].

\section{REFERENCES}

[1] G. Baumslag, M. R. Bridson, C. F. Miller III and H. Short, Fibre products, non-positive curvature, and decision problems, Comment. Math. Helv. 75 (2000), 457-477. MR 2001k:20091

[2] G. Baumslag and J. E. Roseblade, Subgroups of direct products of free groups, J. London Math. Soc. (2) 30 (1984), 44-52. MR 86d:20028

[3] M. Bestvina, M. Handel and M. Feighn, Laminations, trees, and irreducible automorphisms of free groups, Geom. Funct. Anal. 7 (1997), no. 2, 215-244. MR 98c:20045 
[4] N. Brady and A. Miller, CAT(-1) structures for free-by-free groups, Geom. Dedicata 90 (2002), 77-98.

[5] M. R. Bridson, Subgroups of semihyperbolic groups, Monographie de L'Enseign. Math. 38 (2001), 85-111.

[6] M. R. Bridson, The conjugacy and isomorphism problems for combable groups, Math. Ann., to appear.

[7] M. R. Bridson and C. F. Miller III, Finiteness conditions for subdirect products of groups, in preparation.

[8] F. Grunewald, On some groups which cannot be finitely presented, J. London Math. Soc. (2) 17 (1978), 427-436. MR 80d:20033

[9] R. C. Lyndon and P. E. Schupp, "Combinatorial group theory", Springer-Verlag, Berlin, Heidelberg, New York, 1977. MR 58:28182

[10] C. F. Miller III, "On group-theoretic decision problems and their classification", Annals of Mathematics Studies, No. 68, Princeton University Press (1971). MR 46:9147

[11] C. F. Miller III, Decision problems for groups: survey and reflections, in "Algorithms and Classification in Combinatorial Group Theory" (eds. G. Baumslag and C. F. Miller III), MSRI Publications No. 23, Springer-Verlag (1992), 1-59. MR 94i:20057

[12] L. Mosher, A hyperbolic-by-hyperbolic hyperbolic group, Proc. Amer. Math. Soc. 125 (1997), 3447-3455. MR 98c:20069

[13] E. S. Rapaport, Note on Nielsen transformations, Proc. Amer. Math. Soc. 10 (1959), 229235. MR 21:3477

[14] E. Rips, Subgroups of small cancellation groups, Bull. London Math. Soc. 14 (1982), 45-47. MR 83c:20049

Department of Mathematics, 180 Queen's Gate, London SW7 2BZ, United Kingdom

E-mail address: m.bridson@ic.ac.uk

Department of Mathematics and Statistics, University of Melbourne, Parkville 3052, Australia

E-mail address: c.miller@ms.unimelb.edu.au 